\title{
La familia Lauraceae en el sur de México: Diversidad, DISTRIBU CIÓN Y ESTADO DE CONSERVACIÓN
}

\author{
Francisco G. LOREA-HeRnándeZ \\ Instituto de Ecología, A. C., A partado Postal 63, 91000 Xalapa, Veracruz. \\ Correo electrónico: loreaf@ecologia.edu.mx
}

\begin{abstract}
Resumen. Se realizó un análisis general de la diversidad, la distribución y el estado de conservación de la familia Lauraceae en el sur de México. El recuento de la diversidad resultó en una lista de 120 especies comprendidas en 10 géneros: Aiouea, Beilschmiedia, Cassytha, Cinnamomum, Licaria, Litsea, Mocinnodaphne, Nectandra, Ocotea y Persea. Los estados de Chiapas, Oaxaca y Veracruz son las entidades que registran los mayores números de especies, particularmente en el bosque mesófilo de montaña y el bosque tropical perennifolio. De las especies consideradas, $47.5 \%$ son endémicas a México, mismas que se concentran en Chiapas, Oaxaca y Veracruz. La representación de especies de la familia Lauraceae en el sur de México, dentro del sistema de áreas naturales protegidas, es sustancialmente bajo: sólo 58 del total revisado. Se discuten las implicaciones que estos resultados tienen para la perduración de este componente de la flora mexicana en el futuro próximo.

Palabras clave: Lauraceae, México, diversidad, distribución, conservación.

Abstract. A survey of the diversity, distribution, and conservation status of the family Lauraceae in southern Mexico was conducted. The diversity account resulted in a list of 120 species representing 10 genera: Aiouea, Beilschmiedia, Cassytha, Cinnamomum, Licaria, Litsea, Mocinnodaphne, Nectandra, Ocotea and Persea. The states of Chiapas, Oaxaca, and Veracruz have the largest numbers of species, particularly for the cloud forest and the tropical rain forest. Endemic species account for $47.5 \%$ of the surveyed taxa, which concentrate in Chiapas, Oaxaca, and Veracruz. Only 58 of the species considered in the study are present in the system of nature reserves in southern Mexico. The general implications of these findings for the persistence of this component of the Mexican flora are discussed.
\end{abstract}

Key words: Lauraceae, Mexico, diversity, distribution, conservation.

$\mathbf{L}^{2}$ a familia Lauraceae agrupa alrededor de 2500 especies en aproximadamente 50 géneros, distribuidas principalmente en regiones tropicales y subtropicales de todo el mundo (Cronquist, 1981; Rohwer, 1993a). En México no es una familia particularmente diversa, pero está representada por elementos arbóreos importantes de algunas comunidades vegetales, mismas que son interesantes también desde el punto de vista fitogeográfico y por su diversidad biológica (Sarukhán, 1968; Rzedowski, 1978). Tal es el caso de los bosques mesófilos, donde además las Lauraceae han sido identificadas como una fuente importante de sustento para varios grupos de aves (Wheelwright, 1983, 1986; Wheelwright et al., 1984). No obstante su valor biológico, hasta ahora no existe para el país un estudio taxonómico general de la familia, a fin de precisar cuántos taxa verdaderamente existen y conocer cuál es su distribución con más detalle.

De acuerdo con el estudio taxonómico-florístico más reciente que incluye un registro de todas las especies mexicanas de Lauraceae (Allen, 1945), el número de géneros y especies del país se ubicaría en 10 géneros y 73 especies.
Sin embargo, debido a la acumulación y estudio de nuevas colecciones, y la propuesta y formalización de cambios nomenclaturales posteriores, estos datos necesitan ser actualizados. Así, por ejemplo, no obstante que Ocotea es ahora conocido como el género más diverso (43 especies), sólo ocho especies pueden ser ubicadas en ese taxon con base en Allen (1945); a la vez, para Nectandra Rohwer (1993b) considera 18 especies presentes en México, en tanto que Allen (1945) cita 14 (de las cuales varias, en las últimas décadas, han sido reubicadas en Ocotea y algunas como sinónimos de otras especies de Nectandra). Del mismo modo, los estudios que existen ahora para Beilschmiedia (Nishida, 1999), Cinnamomum (Kostermans, 1961; Lorea, 1997), Licaria (Kurz, 2000), Nectandra (Rohwer, 1993b), Ocotea (Rohwer, 1986; van der Werff, 2002a), y Persea (Kopp, 1966; van der Werff, 2002b), ofrecen un mejor conocimiento de las Lauraceae mexicanas. Finalmente, los no pocos taxa nuevos en la familia añadidos a la flora mexicana en los últimos quince años deben ser considerados; véase por ejemplo van der Werff (1987, 1988), Wendt y van der Werff (1987), 
Rohwer (1991), Téllez-Valdés y Villaseñor (1993), Lorea (1995a, b). Tomando en cuenta las modificaciones y enmiendas taxonómicas pertinentes al trabajo de Allen (1945), el número de taxa que pueden ser reconocidos para el país ahora con esa obra se reduce a 7 géneros y 50 especies.

Las Lauraceae gozan de una mala reputación en el sentido de que son taxonómicamente difíciles (Burger, 1988; Rohwer et al., 1991; Gentry, 1993:484-485). Es común encontrar en los herbarios numerosos especímenes asignados a esta familia, sin determinación subsecuente o con una adscripción genérica equívoca. Esto en parte es debido a la confusa delimitación de varios grupos a nivel de género y, por otro lado, al requisito casi imprescindible de tener ejemplares complementarios en flor y fruto para una correcta identificación, lo cual con frecuencia no sucede. Por ello es importante contar con una referencia primaria actualizada que ayude a los botánicos locales en el reconocimiento de las especies existentes de la familia Lauraceae en la región.

Los cambios taxonómicos ocurridos, aunados a la dificultad para determinar los especímenes de esta familia, han conducido a la aplicación de nombres en desuso o equivocados, tornando errática la consulta de varios grupos de especies en nuestros herbarios ( $\mathrm{y}$ en algunos del extranjero también). Esta situación no puede seguir así, ya que la información básica contenida en nuestras colecciones debe ser lo más confiable posible para apoyar otro tipo de estudios, fuera del inventario mismo de nuestros recursos.

Considerando lo anterior, el presente estudio se fijó como meta general sentar las bases sólidas para una revisión taxonómica moderna de la familia Lauraceae en México, analizando de modo crítico la literatura pertinente, así como todo el material de herbario existente en las principales instituciones mexicanas y del extranjero. Los objetivos particulares fueron: (1) producir un catálogo de las especies de las Lauraceae del sur de México, (2) determinar la distribución de las especies, (3) evaluar de modo general el estado de conservación de este importante recurso vegetal, y (4) registrar en una base de datos toda la información disponible de los especímenes conservados en las colecciones revisadas.

La decisión de abordar en el estudio sólo las especies del sur del país se basa en el hecho conocido de la existencia de una mayor diversidad de la familia en esa zona, y a la circunstancia de una división regional específica que la Comisión Nacional para el Conocimiento y Uso de la Biodiversidad (CONABIO), principal financista del trabajo, maneja para la asignación de recursos. Las revisiones taxonómicas correspondientes donde se incluyan descripciones in extenso de los taxa, así como claves adecuadas para su determinación, serán objeto de publicaciones separadas, así como otra adicional con una elaboración más detallada sobre los patrones de distribución de las especies reconocidas.

\section{Método}

La región considerada en este trabajo como el sur de la República Mexicana incluye a los estados de Veracruz, Puebla, Guerrero, Oaxaca, Chiapas, Tabasco, Campeche, Yucatán y Quintana Roo. Como primer paso se recopiló toda la información bibliográfica correspondiente, incluyendo revisiones taxonómicas, floras y publicaciones de nuevos taxa. Al mismo tiempo se solicitaron préstamos de todo el material disponible procedente de la zona de estudio de los herbarios mencionados más adelante. También se visitaron algunos herbarios para separar material directamente y solicitarlo en préstamo, y para revisar especímenes que no son prestados por algunas instituciones. Una vez reunido el material se revisó conforme al siguiente procedimiento: (1) catalogación de los especímenes por género, (2) separación de todas las entidades distinguibles con morfología similar dentro de cada género, (3) análisis en detalle de la morfología vegetativa y reproductora de cada unidad para confirmar la correspondencia entre los individuos, y (4) cotejo de las entidades resultantes con el material tipo. De este modo se buscó precisar los límites y establecer los extremos de variación morfológica de las especies, se detectaron las sinonimias, se reconocieron nuevos taxa, y se estableció la distribución real de las especies en el área. La distinción de las especies hasta ahora se basó en la correlación de caracteres distintivos.

El material estudiado incluyó especímenes de los herbarios CHIP*, CHAPA*, CICY, ECOSUR, ENCB, FCME, MEXU, OAX, UJAT y XAL en México, CAS, F, GH, LL*, MICH, MO, NY y US en Estados Unidos, y B*, $\mathrm{BM}^{*}, \mathrm{BR}, \mathrm{C}^{*}, \mathrm{~K}$ y $\mathrm{P}$ en Europa. Las colecciones de los herbarios marcados con asterisco no fueron revisadas por completo, mas por el estudio de los duplicados distribuidos, no se piensa que haya un porcentaje significativo de colectas diferentes sin estudiar. De todos los tipos consultados durante el trabajo en esas colecciones se tomaron fotografías, para incluirlas en la colección de XAL como material de consulta y referencia para estudios posteriores.

La base de datos se elaboró utilizando el sistema de información BIOTICA 2.0 (CONABIO, 1997), a fin de hacerla compatible con la base de datos del Herbario XAL, que se encuentra organizada con el mismo sistema.

\section{Resultados}

Con base en el material estudiado de los herbarios se llegó a la identificación de 120 especies comprendidas en diez géneros, a saber: Aiouea, Beilschmiedia, Cassytha, Cinnamomum, Licaria, Litsea, Mocinnodaphne, Nectandra, Ocotea y Persea. La relación de las especies reconocidas se presenta en el apéndice 1. En esta lista se consideró pertinente incluir nueve especies y una combinación nuevas ya identificadas que están en proceso de publicación. Si bien 
van der Werff (2002a) considera que Ocotea oblonga (Meisn.) Mez está presente en México, no se encontraron ejemplares correspondientes a esta especie. A fin de ayudar en la correcta determinación del material incorporado en herbarios que no fueron visitados y para el uso de biólogos no especialistas, en el apéndice 2 se anota una lista de los sinónimos más comunes aplicados a las especies tratadas en este estudio.

Los géneros Aniba y Cryptocarya, anteriormente citados por Allen (1945) como presentes en México, en realidad no se encuentran en el país. En el primer caso, Aniba mexicana Kosterm. (Kostermans, 1938) estaba basada en un espécimen de Licaria capitata (Schltdl. et Cham.) Kosterm. de Oaxaca, y en cuanto a Cryptocarya hintonii C.K. Allen (Allen, 1945; que por la ubicación del tipo podría también encontrarse en Guerrero), la descripción se basó en una colecta de Prunus brachybotrya Zucc. (Rosaceae) de Michoacán.

La composición taxonómica de la flora de lauráceas del sur de México presenta varias características interesantes (cuadro 1). En primer término, Ocotea, al igual que en la mayor parte de las floras de la región neotropical, es el género más diverso de la familia. En contraposición, géneros como Aniba, Endlicheria o Pleurothyrium, muy diversos en Sudamérica y con algunos representantes en América Central, no se encuentran en el sur de México, aunque Aiouea, con un patrón de distribución geográfica parecido, sí se halla en la zona. La proporción de especies de los géneros Beilschmiedia, Cinnamomum, Licaria, Nectandra y Persea es más o menos semejante a la de otras floras mesoamericanas. Finalmente, sólo hay un género endémico en la región: Mocinnodaphne, que se conoce en el estado de Guerrero.

El recuento de géneros y especies de lauráceas por entidad federativa (cuadro 2) muestra que los estados de Chiapas, Oaxaca y Veracruz son los más diversos. En cuanto a la

Cuadro 1. N úmero de especies conocidas por género en las Lauraceae del sur de México.

\begin{tabular}{lc}
\hline Género & Número de especies \\
\hline Aiouea & 1 \\
Beilschmiedia & 7 \\
Cassytha & 1 \\
Cinnamomum & 15 \\
Licaria & 17 \\
Litsea & 1 \\
Mocinnodaphne & 1 \\
Nectandra & 19 \\
Ocotea & 43 \\
Persea & 15 \\
\end{tabular}

Cuadro 2. Número de géneros, número total de especies y número de especies endémicas de lauráceas por entidad federativa en el sur de México.

\begin{tabular}{lccc}
\hline Estado & Géneros & Especies & $\begin{array}{c}\text { Especies } \\
\text { Endémicas }\end{array}$ \\
\hline Campeche & 3 & 7 & 0 \\
Chiapas & 9 & 77 & 22 \\
Guerrero & 8 & 32 & 15 \\
O axaca & 8 & 68 & 27 \\
Puebla & 7 & 15 & 6 \\
Q uintana Roo & 4 & 7 & 0 \\
Tabasco & 5 & 21 & 1 \\
Veracruz & 9 & 55 & 19 \\
Yucatán & 3 & 4 & 0 \\
\hline
\end{tabular}

presencia de taxa de la familia en los tipos de vegetación (sensu Rzedowski, 1978) de la zona de estudio (cuadro 3), los bosques mesófilos de montaña y el bosque tropical perennifolio son los más ricos. En este punto puede notarse, en general, que a menor disponibilidad de agua se corresponde un descenso en la diversidad de esta familia en el sur de México.

Las especies mejor representadas en los herbarios (con más de 130 y hasta 350 colectas en total por especie) son Litsea glaucescens, Nectandra coriacea, N. salicifolia, Ocotea helicterifolia y Persea americana. El resto de las especies cuentan con 60 o menos números colectados. Cerca de cuarenta especies se conocen de entre 35 y 10 colectas diferentes, y un número semejante de especies están ejemplificadas por entre una y cinco colectas.

Cuadro 3. Número de especies conocidas de Lauraceae por tipo de vegetación en el sur de México.

\begin{tabular}{lc}
\hline Tipo de vegetación & Especies de Lauraceae \\
\hline Bosque Tropical Perennifolio & 51 \\
Bosque Tropical Subcaducifolio & 29 \\
Bosque Tropical Caducifolio & 10 \\
Bosque de Coníferas & 9 \\
Bosque de Encino (Encino-Pino) & $26(25)$ \\
Bosque M esófilo de Montaña & 73 \\
Pastizal & 4 \\
Manglar & 1 \\
\hline
\end{tabular}


Cuadro 4. Distribución geográfica de las especies de lauráceas del sur de México.

\begin{tabular}{llc}
\hline Distribución & \multicolumn{2}{c}{$\begin{array}{l}\text { Número de especies } \\
\text { (porcentaje del total) }\end{array}$} \\
\hline Endémicas & 57 & $(47.5 \%)$ \\
Sur de México - Honduras & 32 & $(26.6 \%)$ \\
Sur de México - Panamá & 14 & $(11.6 \%)$ \\
Sur de México - Sudamérica & 7 & $(5.8 \%)$ \\
Sur de México - Sudamérica - & 7 & $(5.8 \%)$ \\
Las Antillas & & \\
Sur de México - Las Antillas & 3 & $(2.5 \%)$ \\
\hline
\end{tabular}

El análisis de la distribución geográfica de las especies de Lauraceae consideradas en este trabajo (cuadro 4), revela algunos rasgos significativos: (1) $47.5 \%$ de las especies son endémicas a México, o hasta 74\% si se toma el área sugerida por Rzedowski (1991) como Megaméxico 2 (extendiendo la frontera sur hasta el norte de Nicaragua), (2) en consecuencia, hay muy pocas especies de distribución amplia en América representadas en el área de estudio, (3) la relación con la flora antillana se da tanto por especies de amplia distribución como por algunas sólo compartidas con el sur de México, (4) no existen especies de distribución anfitropical o mundial en esta muestra de la flora mexicana.

Cabe señalar que con base en el material estudiado, aquí se considera a Ocotea acuminatissima como endémica, en tanto que a $O$. parvula, $O$. purpurea y $O$. verticillata como presentes, las dos primeras, solamente en Chiapas y sólo en Veracruz la última; esto resulta en pequeñas diferencias con la distribución que cita van der Werff (2002a) para estas especies. Como el origen de estas divergencias pueden deberse a determinaciones distintas de los mismos ejemplares, se optó por no considerar esos datos. Como sea, estas discrepancias no alteran las conclusiones que más adelante se exponen.

Tomando sólo el elemento endémico a México (cuadro 2 ), se encuentra que éste alcanza niveles relativamente altos en Chiapas, Oaxaca y Veracruz, con 22 (38.6\%), 27 (47.3\%) y 19 (33.3\%) especies endémicas del total de este grupo, respectivamente. Guerrero tiene un valor más moderado $(26.3 \%, 15$ especies) y Puebla una cifra baja $(10.5 \%, 6$ especies). Resulta interesante indicar en el caso de Guerrero y Puebla que, no obstante su baja representación del endemismo global (hablando de las lauráceas mexicanas), localmente tienen una proporción más alta $(46.8 \%$ y $40 \%$, respectivamente) que los tres estados más ricos en especies endémicas de esa familia. En Tabasco se conoce hasta ahora una sola especie endémica, en tanto que en Campeche, Quintana Roo y Yucatán no se encuentra ninguna especie endémica.
Cuadro 5. Número de especies conocidas de Lauraceae en áreas naturales protegidas del sur de México.

\begin{tabular}{ll}
\hline Área natural protegida & Número de especies \\
\hline Calakmul (Campeche) & 1 \\
Bonampak (Chiapas) & 12 \\
Cañón del Sumidero (Chiapas) & 6 \\
Cascadas de Agua Azul (Chiapas) & 1 \\
El Triunfo (Chiapas) & 16 \\
Lacan-Tun (Chiapas) & 15 \\
Lagunas de Montebello (Chiapas) & 7 \\
Montes Azules (Chiapas) & 2 \\
Palenque (Chiapas) & 15 \\
Yaxchilán (Chiapas) & 8 \\
El Veladero (Guerrero) & 2 \\
Grutas de Cacahuamilpa (Guerrero) & 1 \\
Benito Juárez (O axaca) & 10 \\
Huatulco (O axaca) & 1 \\
Lagunas de Chacahua (O axaca) & 1 \\
Tehuacán-Cuicatlán (Puebla-O axaca) & 19 \\
Tulum (Q uintana Roo) & 5 \\
Cañón de Río Blanco (Veracruz) & 1 \\
Cofre de Perote (Veracruz) & 2 \\
Los Tuxtlas (Veracruz) & 1 \\
Pico de O rizaba (Puebla-Veracruz) & \\
Ría Celestún (Campeche-Yucatán) & \\
& \\
\hline
\end{tabular}

Por lo que toca a las especies de lauráceas presentes en las diferentes áreas naturales protegidas de tipo federal (Reserva de la Biosfera, Parque Nacional, Monumento Natural, Áreas de Protección de Flora y Fauna, o de Recursos Naturales), al parecer en 22 (Cuadro 5) de las 40 áreas terrestres existentes en el sur de México se encuentran registros de especies de esa familia de plantas (conforme a la base de datos elaborada). La incertidumbre del dato, en este caso, se debe a la falta de información acerca de los límites geográficos de las áreas, imprecisión en los datos de colecta de los especímenes o una combinación de ambas situaciones. Como sea, hasta ahora solamente un total de 58 especies de las 120 reconocidas para el área de estudio se han registrado de alguna de las áreas naturales protegidas. De ellas, 14 son endémicas, 21 se encuentran del sur de México a Honduras, 8 se distribuyen hasta Panamá, 6 hasta Sudamérica, 5 tienen una distribución en toda la zona tropical americana incluyendo Las Antillas, y una especie se halla entre el sur de México y Las Antillas. 


\section{Discusión}

Las 120 especies reconocidas en el presente trabajo representan alrededor de $90-93 \%$ de la diversidad total de las lauráceas mexicanas. Se conocen con seguridad otras ocho especies para el resto del país (una Persea, cuatro Cinnamomum, dos Litsea y una Beilschmiedia), más otras dos posiblemente nuevas. Con ello, existen ahora las bases para elaborar en el corto plazo una revisión taxonómica general (ya en proceso) de la familia Lauraceae para México.

Los datos hasta ahora obtenidos muestran que la diversidad de la familia Lauraceae en México es más grande que lo considerado hace cincuenta años (Allen, 1945); cerca de $86 \%$ más que lo estimado en ese tiempo (o $140 \%$ si se toman en cuenta los ajustes taxonómicos necesarios en ese trabajo). El desconocimiento prevaleciente de las lauráceas mexicanas no sólo es consecuencia de un reiterado estudio deficiente de la familia, taxonómicamente hablando, sino también de la escasa representación de la flora de ciertas áreas de México en las colecciones de los herbarios. De las cerca de 2800 colectas diferentes revisadas para este trabajo, casi $60 \%$ son colectas efectuadas en los últimos 25 años. Esto tiene que ver, de alguna manera, con la descripción de 25 especies de lauráceas mexicanas en ese mismo intervalo de tiempo (más las nueve adicionales que están en proceso de publicación).

A pesar de este repunte en las colectas de lauráceas en el sur de México, la familia aún está en general mal representada en los herbarios. El esfuerzo de colecta, medido como el cociente "número de colectas / número de especies", muestra que Chiapas, Quintana Roo y Veracruz, con valores de 13.9, 19.7 y 11.5 , respectivamente, son los estados mejor colectados (en lo que toca a las Lauraceae). Tabasco y Guerrero, con valores cercanos a 3 , al parecer serían los peor colectados. Las entidades restantes, Campeche, Oaxaca, Puebla y Yucatán, tienen valores de entre 6 y 8 . Estas cifras, al igual que los datos de número de colectas por especie referidos en la sección anterior, sugieren que es necesaria una exploración más exhaustiva en los restos de vegetación natural (protegidos o no) de varios estados, a fin de tener una idea mejor de la distribución de las especies de lauráceas a escalas regional o local en esta zona del país.

El alto porcentaje de endemismo hallado en las lauráceas de México (50\% si se toman en cuenta todas las especies) es equiparable al que tienen otras familias de angiospermas en el país, como Acanthaceae (ca. 50\%; Daniel, 1993), Commelinaceae (50\%; Hunt, 1993), Lamiaceae (ca. 77\%; Ramamoorthy y Elliot, 1993) y Leguminosae (ca. 52\%; Sousa y Delgado, 1993), entre otras. El dato refuerza la peculiaridad de la flora mexicana y remarca, una vez más, la importancia de nuestros recursos vegetales por su significado biológico. En este punto, es interesante anotar que los géneros Cinnamomum, Ocotea y Persea, que exhiben una afinidad por hábitats donde prospera el bosque mesófilo de montaña, tienen los valores más altos de endemismo $(73 \%, 53.5 \%$ y $66.6 \%$, respectivamente). Por su parte, Beilschmiedia y Licaria, que se encuentran más o menos igualmente presentes en ambientes de tipo templado o cálido, tienen una tasa de endemismo de $28.6 \%$ y $47 \%$, respectivamente. Aiouea, Cassytha y Nectandra, todos ellos con clara afinidad por ambientes cálidos, no presentan especies endémicas (los dos primeros) o valores bajos (10.5\%, el último) de endemismo. El género Litsea, de afinidad templada, no posee especies endémicas en el sur de México, pero a nivel nacional llega al $66 \%$ de endemismo. En resumen, es probable que el origen del patrón de reparto del endemismo en las lauráceas esté vinculado no propiamente con un hábitat particular, sino en cómo se distribuye éste (continuo o fragmentado).

Salvo algunas excepciones, como por ejemplo Cinnamomum effusum, Ocotea acuminatissima, $O$. betazensis, O. psychotrioides y Persea liebmanni, muchas de las especies endémicas son al parecer localmente escasas o poco abundantes. Al menos 25 de ellas se conocen por cinco o menos colectas, lo que puede en cierta manera dar idea de lo reducido del área que ocupan varias de estas especies en la geografía nacional.

De cualquier modo, es claro que, en contraposición con el patrón general de distribución del endemismo en México (Rzedowski, 1991), que se concentra en las zonas áridas, las lauráceas no tienen presencia en este tipo de ambientes. Por el contrario, la mayor diversidad y endemismo de la familia se encuentra en áreas más bien húmedas; de las 73 especies que habitan el bosque mesófilo, 40 (casi 55\%) son endémicas, y de éstas, 23 sólo se conocen de este tipo de vegetación.

De las especies mejor representadas en los herbarios no hay indicios, excepto de alguna manera para Nectandra salicifolia, que su amplia distribución puede ser favorecida por el disturbio. Al parecer, tanto ésta como las otras especies con muchas colectas son en realidad más abundantes y dispersas en la naturaleza. La amplia representación de Persea americana está influida, aunque no sustancialmente, por su introducción a varios lugares por el consumo de sus frutos. De cualquier forma, el patrón observado del número de colectas por especie en su conjunto revela la singularidad de esta porción de la flora mexicana; es un hecho que muchas de las especies están restringidas a hábitats y áreas reducidas, incluso en el caso de especies no endémicas.

El número de especies registradas por tipo de vegetación resalta la importancia de conservar los pocos remanentes de bosque mesófilo de montaña y bosque tropical perennifolio que subsisten en México. Como se muestra, estas comunidades vegetales albergan, al igual que para otros taxa, la mayor parte de la diversidad de Lauraceae. Sin embargo, es interesante señalar que el bosque mesófilo podría tener una significación mayor para la conservación de la diversidad de esta familia de plantas en México, ya que contiene un mayor número de endemismos (40 especies de esta clase), que el bosque tropical perennifolio (con sólo 10 especies 
endémicas). Esta situación implica también la influencia que ha tenido la historia de las comunidades de bosque mesófilo (su distribuciónn fragmentada) en la evolución de la diversidad de esta familia en México.

En relación al punto anterior, preocupa el hecho que hasta hoy existen sólo dos áreas naturales protegidas de carácter federal que tienen una buena representación de bosque mesófilo: El Triunfo (Chiapas) y Los Tuxtlas (Veracruz) que, cabe destacar, son las áreas con mayor número de lauráceas en su flora. Considerando la distribución del bosque mesófilo, hace falta y urge establecer áreas protegidas que incluyan este tipo de vegetación en los estados de Guerrero, Oaxaca y Puebla.

Los datos del recuento de especies de lauráceas en las áreas naturales protegidas aquí examinadas son preliminares, debido a que en general no existen estudios florísticos acabados para la mayor parte de ellas. De cualquier forma, es muy probable que la baja representación de la diversidad de lauráceas (casi 50\% de las especies no están presentes) no disminuya en modo apreciable aun realizando estudios florísticos completos de todas las áreas. La distribución de las especies endémicas determina la necesidad de establecer áreas en otros sitios (sobre todo en el estado de Oaxaca) para incrementar las posibilidades de conservación de una parte sustancial de la diversidad de lauráceas en el sur de México.

La información hasta ahora reunida no permite determinar, con certeza, el estado de riesgo en que se encuentran las poblaciones de las diferentes especies de Lauraceae del sur de México. No obstante, en general se puede decir que dado el grado de deterioro de las comunidades vegetales de esta parte del país, las dimensiones de las áreas de distribución de las especies consideradas, y la baja representación de los taxa referidos en áreas naturales protegidas, es probable que cerca de la mitad de las especies se encuentre bajo amenaza de extinción. El reducido número de colectas (mencionados en la sección de resultados) de muchas de las especies puede ser significativo en ese sentido. Considerando algunos de estos datos, es posible que Cinnamomum amplexicaule, C. concinnum, Licaria chinanteca, Nectandra leucocome, Ocotea iridescens, $O$. corrugata y Persea longipes (entre otras) se hallen en peligro de extinción, en tanto que Licaria mexicana y Ocotea subalata podrían estar ya extintas. Debido al hecho de que la gran mayoría de las especies de esta familia no prosperan en vegetación secundaria, las torna muy vulnerables al disturbio, por lo que la situación de subsistencia podría ser crítica para muchas de ellas.

El corolario a la exposición de los datos presentados en este estudio sería que la revisión similar de otros taxa de la flora de México ayudaría a configurar una mejor imagen de la dimensión de su diversidad, de su arreglo en el paisaje del país y, particularmente, a crear una base más sólida para conocer el estado que guarda su conservación. Idealmente, la suma de varios estudios de este tipo posibilitarían una mejor estrategia para preservar lo más posible de nuestra rica e interesante flora. Nunca como ahora los trabajos de revisión sistemática deben proporcionar, al mismo tiempo que la información biológica pertinente, los datos primarios para conservar su objeto de estudio.

\section{Agradecimientos}

La elaboración del presente trabajo contó con un apoyo sustancial de la CONABIO, mediante el convenio FB517/ L090/97. Se agradece la colaboración de la Biól. Eva Pérez y la L. I. Maricruz Peredo en la construcción, reformas reiteradas y manejo de la base de datos en que se basa este estudio. La revisión crítica y atinada del texto por parte de Jerzy Rzedowski, Thomas L. Wendt y un revisor anónimo ayudaron a mejorar la presentación del trabajo. La labor editorial de Jorge Meave ha sido inmejorable.

\section{Literatura citada}

Allen C.K. 1945. Studies in the Lauraceae VI. Preliminary survey of the Mexican and Central American species. Journal of the Arnold Arboretum 26:280-434.

Burger W.C. 1988. A new genus of Lauraceae from Costa Rica, with comments on problems of generic and specific delimitation within the family. Brittonia 40:275-282.

CONABIO (Comisión Nacional para el Conocimiento y Uso de la Biodiversidad) 1997. Sistema de Información Biótica 2.0, CONABIO, México, D.F.

Cronquist A. 1981. An Integrated System of Classification of Flowering Plants. Columbia University Press. Nueva York.

Daniel T.F. 1993. Mexican Acanthaceae: diversity and distribution. En: Ramamoorthy T.P., Bye R., Lot A. y Fa J. Eds. Biological Diversity of Mexico. Origins and Distribution, pp. 541-558. Oxford University Press, Nueva York.

Gentry A.H. 1993. A Field Guide to the Families and Genera of Woody Plants of Northwest South America (Colombia, Ecuador, Peru) with Supplementary Notes on Herbaceous Taxa. The University of Chicago Press, Chicago.

Hunt D.R. 1993. The Commelinaceae of Mexico. En: Ramamoorthy T.P., Bye R., Lot A. y Fa J. Eds. Biological Diversity of Mexico. Origins and Distribution, pp. 421-437, Oxford University Press, Nueva York.

Kopp L.E. 1966. A taxonomic revision of the genus Persea in the western hemisphere (Perseae-Lauraceae). Memoirs of the New York Botanical Garden 14:1-120.

Kostermans A.J.G.H. 1938. Revision of the Lauraceae V. A monograph of the genera: Anaueria, Beilschmiedia (American species) and Aniba. Recueil des Travaux Botaniques Néerlandais 35:834-931.

Kostermans A.J.G.H. 1961. The new world species of Cinnamomum Trew. (Lauraceae). Reinwardtia 6:17-24.

Kurz H. 2000. Revision der Gattung Licaria (Lauraceae). Mitteilungen aus dem Institut für Allgemeine Botanik in Hamburg 28/29:89-221.

Lorea F. 1995a. A new species of Beilschmiedia (Lauraceae) from Guerrero, Mexico. Novon 5:45-47. 
Lorea F. 1995b. Mocinnodaphne, un género nuevo de la familia Lauraceae en la flora de México. Acta Botanica Mexicana 32:2532.

Lorea F. 1997. On Cinnamomum (Lauraceae) in Mexico. Acta Botanica Mexicana 40:1-18.

Nishida S. 1999. Revision of Beilschmiedia (Lauraceae) in the Neotropics. Annals of the Missouri Botanical Garden 86:657701.

Ramamoorthy T.P. y Elliot M. 1993. Mexican Lamiaceae: diversity, distribution, endemism, and evolution. En: Ramamoorthy T.P., Bye R., Lot A. y Fa J. Eds. Biological Diversity of Mexico. Origins and Distribution, pp. 513-539. Oxford University Press, Nueva York.

Rohwer J.G. 1986. Prodromus einer Monographie der Gattung Ocotea Aubl. (Lauraceae) sensu lato. Mitteilungen aus dem Institut für Allgemeine Botanik in Hamburg 20:1-278.

Rohwer J.G. 1991. Borderline cases between Ocotea, Nectandra, and Phoebe (Lauraceae): the "marginal" species of the Ocotea helicterifolia-group, including the $O$. heydeana-group. Botanische Jahrbücher für Systematik 112:365-397.

Rohwer J.G. 1993a. Lauraceae. En: Kubitzki K. Ed. The Families and Genera of Vascular Plants. Vol. 2, pp. 366-391, SpringerVerlag, Nueva York.

Rohwer J.G. 1993b. Lauraceae: Nectandra. Flora Neotropica Monograph 60:1-333.

Rohwer J.G., Richter H.G. y van der Werff H. 1991. Two new genera of neotropical Lauraceae and critical remarks on the generic delimitation. Annals of the Missouri Botanical Garden 78:388400.

Rzedowski J. 1978. Vegetación de México. Limusa. México, D.F.

Rzedowski J. 1991. Diversidad y orígenes de la flora fanerogámica de México. Acta Botanica Mexicana 14:3-21.

Sarukhán J. 1968. Los tipos de vegetación arbórea de la zona cálidohúmeda de México. En: Pennington T.D. y Sarukhán J. Eds.
Manual para la Identificación de los Principales Árboles Tropicales de México, pp. 3-46, Instituto Nacional de Investigaciones Forestales y FAO, México, D.F.

Sousa M. y Delgado A. 1993. Mexican Leguminosae: phytogeography, endemism, and origins. En: Ramamoorthy T.P., Bye R., Lot A. y Fa J. Eds. Biological Diversity of Mexico. Origins and Distribution, pp. 459-511, Oxford University Press, Nueva York.

Téllez-Valdés O. y Villaseñor J.L. 1993. Cinnamomum kruseanum (Lauraceae), a new species from Guerrero, México. Novon 3:208-210.

van der Werff H. 1987. Six new species of neotropical Lauraceae. Annals of the Missouri Botanical Garden 74:401-412.

van der Werff H. 1988. Eight new species and one new combination of neotropical Lauraceae. Annals of the Missouri Botanical Garden 75:402-419.

van der Werff H. 2002a. A synopsis of Ocotea (Lauraceae) in Central America and southern Mexico. Annals of the Missouri Botanical Garden 89:429-451.

van der Werff H. 2002b. A synopsis of Persea (Lauraceae) in Central America. Novon 12:575-586.

Wendt T. y van der Werff H. 1987. A new species of Ocotea (Lauraceae) from southeastern Mexico. Annals of the Missouri Botanical Garden 74:413-415.

Wheelwright N.T. 1983. Fruits and the ecology of resplendent quetzals. The Auk 100:286-301.

Wheelwright N.T. 1986. A seven-year study of individual variation in fruit production in tropical bird-dispersed tree species in the family Lauraceae. En: Estrada A. y Fleming T.H. Eds. Frugivores and Seed Dispersal, pp. 19-35, Dr. W. Junk, Dordrecht.

Wheelwright N.T., Haber W.A., Murray K.G. y Guindon C. 1984. Tropical fruit-eating birds and their food plants: a survey of a Costa Rican lower montane forest. Biotropica 16:173-192. 
A péndice 1. Especies de Lauraceae del sur de M éxico, su distribución por estados y tipo de vegetación donde crecen. Distribución: $\mathrm{V}=$ Veracruz, $\mathrm{P}=$ Puebla, $\mathrm{G}=\mathrm{G}$ uerrero, $\mathrm{O}=\mathrm{O}$ axaca, $\mathrm{Ch}=$ Chiapas, $\mathrm{T}=$ Tabasco, $\mathrm{Ca}=$ Campeche, $\mathrm{Y}=$ Yucatán, $\mathrm{Q} \mathrm{R}=\mathrm{Q}$ uintana Roo. $X=$ nativa, $C=$ cultivada. Tipo de vegetación: $b c=$ bosque de coníferas, $b e=$ bosque de encino, $b m m=$ bosque mesófilo de montaña, $b p e=$ bosque de pino-encino, $b t c=$ bosque tropical caducifolio, $b t p=$ bosque tropical perennifolio, $b t s c=$ bosque tropical subcaducifolio, $m=$ manglar, $p=$ pastizal. Las especies endémicas de México se anotan en negritas.

\begin{tabular}{|c|c|c|c|c|c|c|c|c|c|c|}
\hline & V & $\mathrm{P}$ & G & 0 & $\mathrm{Ch}$ & $\mathrm{T}$ & $\mathrm{Ca}$ & Y & QR & \\
\hline \multicolumn{11}{|l|}{ AIOUEA } \\
\hline A. inconspicua van der Werff & $x$ & & & $\mathrm{x}$ & $\mathrm{X}$ & $\mathrm{x}$ & & & & btsc, btp \\
\hline \multicolumn{11}{|l|}{ BEILSCHMIEDIA } \\
\hline B. anay (S.F.Blake) Kosterm. & $\mathrm{x}$ & & & $\mathrm{x}$ & & & & & & bmm, btsc \\
\hline B. angustielliptica Lorea-Hern. & & & $\mathrm{x}$ & & & & & & & bmm \\
\hline B. hondurensis Kosterm. & $\mathrm{x}$ & & & $\mathrm{x}$ & $\mathrm{x}$ & & & & & bmm, bpe, btp \\
\hline B. mexicana (M ez) Kosterm. & $x$ & $\mathrm{x}$ & & & & & & & & bmm \\
\hline B. ovalioides Sa.N ishida & & & & $\mathrm{x}$ & $\mathrm{x}$ & & & & & $\mathrm{bmm}$ \\
\hline B. ovalis (S.F Blake) C.KAllen & & & & $\mathrm{x}$ & $\mathrm{x}$ & & & & & be, bmm \\
\hline B. riparia Miranda & & & $\mathrm{x}$ & $\mathrm{x}$ & $\mathrm{x}$ & & & & & be, btp, btsc \\
\hline \multicolumn{11}{|l|}{ CASSYTHA } \\
\hline C. filiformis L. & $\mathrm{x}$ & & & & $x$ & & $x$ & $x$ & $x$ & $\begin{array}{l}\text { be, btc, btp, } \\
\text { btsc, m, p }\end{array}$ \\
\hline \multicolumn{11}{|l|}{ CINNAMOMUM } \\
\hline C. amplexicaule (Schltdl. et Cham.) Kosterm. & $\mathrm{X}$ & & $\mathrm{x}$ & $\mathrm{X}$ & & & & & & be \\
\hline C. areolatum (Lundell) Kosterm. & & & & $\mathrm{x}$ & $\mathrm{x}$ & & & & & $\mathrm{bc}, \mathrm{be}, \mathrm{bmm}$ \\
\hline C. breedlovei (Lundell) Kosterm. & & & & $x$ & $x$ & & & & & bmm \\
\hline C. chiapense (Lundell) Kosterm. & & & & & $\mathrm{x}$ & & & & & bc \\
\hline C. concinnum Lorea-Hern. & & & & $\mathrm{x}$ & & & & & & bmm \\
\hline C. effusum (M eissn.) Kosterm. & $\mathrm{x}$ & $x$ & & & & & & & & be, bmm \\
\hline C. grisebachii Lorea-H ern. & & & & $\mathrm{x}$ & $\mathrm{x}$ & $\mathrm{x}$ & & & & btp \\
\hline C. kruseanum Villaseñor et 0 . Téllez & & & $\mathrm{x}$ & & & & & & & bpe \\
\hline C. leptophyllum Lorea-H ern. & $\mathrm{x}$ & & & & & & & & & be, bmm \\
\hline C. pachypodum (N ees) Kosterm. & $x$ & $\mathrm{X}$ & & & & & & & & $\mathrm{bmm}$ \\
\hline C. padiforme (Standl. et Steyerm.) Kosterm. & & & $\mathrm{x}$ & & & & & & & $\mathrm{bmm}$ \\
\hline C. triplinerve (Ruiz et Pav.) Kosterm. & $x$ & & $\hat{x}$ & $x$ & $x$ & $x$ & & & & bpe, btp, btsc \\
\hline C. velveti Lorea-Hern. & & & $\mathrm{x}$ & & & & & & & bpe \\
\hline C. zapatae Lorea-Hern. & & & $\mathrm{x}$ & $\mathrm{x}$ & $\mathrm{x}$ & & & & & $\mathrm{bmm}$ \\
\hline C. sp. nov. & & & & & $\mathrm{x}$ & & & & & $\mathrm{bmm}$ \\
\hline \multicolumn{11}{|l|}{$\begin{array}{l}\text { LICARIA } \\
\text { LIS }\end{array}$} \\
\hline L. brittoniana C.K.Allen et L.E.Gregory & & & & $\mathrm{X}$ & & & & & & btc \\
\hline L. campechiana (Standl.) Kosterm. & $\mathrm{x}$ & & & & $\mathrm{x}$ & & $\mathrm{x}$ & $\mathrm{x}$ & $\mathrm{x}$ & btc, btp, btsc \\
\hline L. capitata (Schltdl. et Cham.) Kosterm. & $x$ & $x$ & & $x$ & $x$ & $x$ & & & & $\begin{array}{l}\text { be, bmm, bpe, } \\
\text { btc, btp, btsc }\end{array}$ \\
\hline L. caudata (Lundell) Kosterm. & & & & & $\mathrm{x}$ & & $\mathrm{x}$ & & & btc, btp, btsc \\
\hline L. cervantesii (H.B.K.) Kosterm. & & & $\mathrm{x}$ & $\mathrm{x}$ & $\mathrm{x}$ & $\mathrm{x}$ & & & & btp, btsc \\
\hline L. chinanteca Lorea-H ern. & & & & $\mathrm{x}$ & & & & & & $\mathrm{bmm}$ \\
\hline L. excelsa Kosterm. & $x$ & & $x$ & $\hat{x}$ & $x$ & & & & & bmm, btp, btsc \\
\hline L. glaberrima (Lundell) C.K.Allen & & & & & $\mathrm{x}$ & & & & & bmm, bpe \\
\hline L. mexicana (Brandegee) Kosterm. & $\mathrm{x}$ & & & & & & & & & bmm? \\
\hline L. misantlae (Brandegee) Kosterm. & $x$ & & & & $x$ & & & & & be, btc, btp, btsc \\
\hline L. multinervis Kurz & & & & & $\mathrm{X}$ & & & & & bmm-bpe \\
\hline $\begin{array}{l}\text { L. peckii (I.M.Johnst.) Kosterm. } \\
\text { L. phymatosa nom. ined. }\end{array}$ & $\mathrm{X}$ & & $\mathrm{X}$ & $\mathrm{X}$ & $\mathrm{X}$ & $\mathrm{X}$ & $\mathrm{X}$ & & $\mathrm{X}$ & $\begin{array}{l}\text { be, bmm, bpe, btp, btsc } \\
\text { bmm }\end{array}$ \\
\hline L. quercioidea nom. ined. & & & $x$ & & & & & & & be \\
\hline L. siphonantha nom. ined. & & & $\mathrm{x}$ & & & & & & & bmm \\
\hline L. urceolata Lundell & & & $x$ & $\mathrm{X}$ & & & & & & bpe \\
\hline L. velutina van der Werff & $\mathrm{x}$ & & & $x$ & $\mathrm{x}$ & & & & & btp \\
\hline LITSEA & & & & & & & & & & \\
\hline L. glaucescens H.B.K. & $x$ & $\mathrm{X}$ & $\mathrm{X}$ & $\mathrm{X}$ & $\mathrm{x}$ & & & & & bc, be, bmm, bpe, btc \\
\hline
\end{tabular}




\section{MOCINNODAPHNE}

M. cinnamomoidea Lorea-Hern. NECTANDRA

$N$. ambigens (S.F.Blake) C.K.Allen

$N$. cissiflora $\mathrm{N}$ ees

$N$. colorata Lundell

N. coriacea (Sw.) Griseb.

$N$. cuspidata $\mathrm{N}$ ees

$N$. hihua (Ruiz et Pav.) Rohwer

$\boldsymbol{N}$. leucocome Rohwer

$N$. longicaudata (Lundell) C.K.Allen

$N$. lundellii C.K.Allen

N. martinicensis M ez

$N$. matudai Lundell

N. membranacea (Sw.) G riseb.

N. nitida Mez

$N$. purpurea (Ruiz et Pav.) Mez

$N$. reticulata (Ruiz et Pav.) Mez

$N$. rudis C.K.Allen

N. salicifolia (H.B.K.) N ees

N. salicina C.K.Allen

N. turbacensis (H.B.K.) N ees

OCOTEA

O. acuminatissima (Lundell) Rohwer

0 . bernoulliana $\mathrm{M} \mathrm{ez}$

$\boldsymbol{0}$. betazensis (M ez) van der Werff

0 . botrantha Rohwer

0 . bourgeauviana (M ez) van der Werff

0 . candidovillosa Lorea-Hern.

O. cernua ( $\mathrm{N}$ ees) M ez

O. chiapensis (Lundell) Standl. et Steyerm.

0 . chrysobalanoides (Lundell) Lundell

0 . congregata van der Werff

$\boldsymbol{0}$. corrugata van der Werff

0 . dendrodaphne $\mathrm{Mez}$

o. disjuncta Lorea-H ern.

0 . divaricata nom. ined.

0 . effusa (M eissn.) Hemsl.

0 . helicterifolia (M eissn.) Hemsl.

0 . heribertoi Wendt

$\boldsymbol{0}$. iridescens Lorea-H ern. et van der Werff

O. klotzschiana (N ees) Hemsl.

0 . laetevirens Standl. et Steyerm.

0 . leucoxylon (Sw.) M ez

O. magnifolia (Lundell) Lundell

0 . matudai Lundell

0. parvula (Lundell) van der Werff

O. platyphylla (Lundell) Rohwer

0 . psychotrioides H.B.K.

0 . puberula (Rich.) N ees

0 . purpurea (Mez) van der Werff

o. rovirosae Lorea-H ern. et van der Werff

0 . rubriflora $\mathrm{Mez}$

0 . salvinii M ez

0 . sarcodes nom. ined.
X

bmm

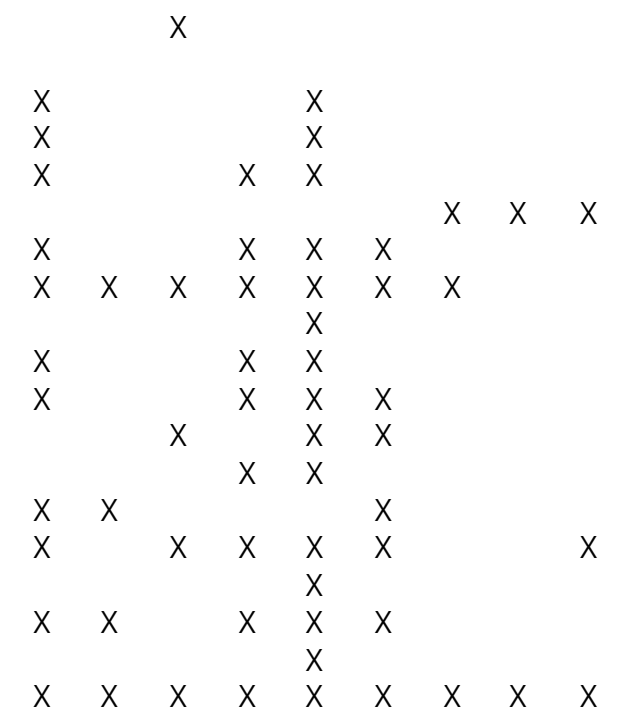

btp

btp

btp

btp, btsc, p

bmm, btp, btsc

bpe, btp, btsc

btp

btp, btsc

btp

btp, btsc

be, bpe

btp

btp, btsc

btsc?

be, bmm, btp, btsc, p

bmm

be, bmm, bpe,

btc, btp, btsc

bmm

btc, btp

bmm

bmm, bpe, btp, btsc

bc, be, bmm

bmm, bpe, btp, btsc

bmm, btp, btsc

bmm

btp, btsc

bmm

bmm?

bmm, bpe

btsc

bmm, btp, btsc

bmm

bmm, be

bmm, btp

bc, be, bmm, bpe, btp, btsc

btp

bmm, bpe

be, bmm, bpe

btp

bmm, btp

btp

bmm

bmm

bmm

be, bmm

btp

bc, bmm

btp

btp

bmm, bpe

bmm 


\section{$\begin{array}{llllllllll}V & P & G & O & C h & T & C a & Y & Q R\end{array}$}

O. sauroderma Lorea-H ern.

O. sinuata (M ez) Rohwer

O. standleyi C. K. Allen

O. subalata Lundell

O. tampicensis (M eissn.) Hemsl.

$\boldsymbol{O}$. tonii (Lundell) van der Werff

0 . truncata Lundell

o. uxpanapana W endt et van der Werff

0 . veraguensis (M eissn.) M ez

0 . verticillata Rohwer

$\boldsymbol{O}$. zoque nom. ined.

\section{PERSEA}

P. albida Kosterm.

P. americana Mill.

P. brevipetiolata van der Werff

P. chamissonis M ez

P. chrysantha nom. ined.

P. cinerascens S.F.Blake

P. donnell-smithii M ez

P. hintonii C.K.Allen

P. liebmannii M ez

P. longipes (Schltdl.) Meissn.

P. obscura nom. ined.

P. pallescens nom. ined.

$\boldsymbol{P}$. rufescens Lundell

$P$. schiedeana $\mathrm{N}$ ees

$P$. vesticula Standl. et Steyerm.

$\begin{array}{ll}X & \\ X & X \\ & X \\ & X\end{array}$

$X$

$\begin{array}{llll} & x & X \\ x & & & X \\ x & x & X \\ & & & \end{array}$

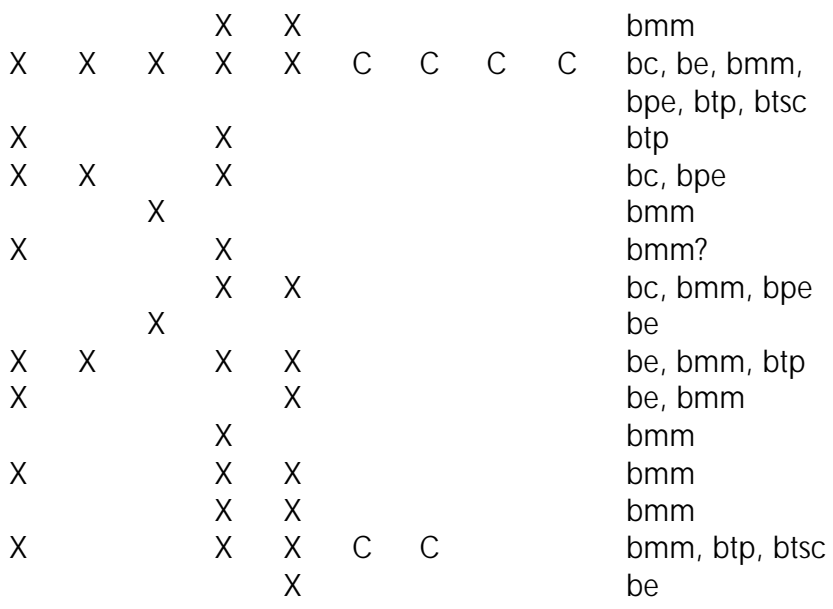


Apéndice 2. Sinónimos (taxonómicos y nomenclaturales) más comúnmente aplicados a las Lauraceae del sur de México.

Beilschmiedia anay: Hufelandia anay S.F.Blake

Beilschmiedia mexicana: Hufelandia mexicana M ez, Linociera areolata Lundell

Beilschmiedia ovalis: Hufelandia ovalis S.F.Blake

Cinnamomum amplexicaule: Persea amplexicaulis Schltdl. et Cham., Phoebe amplexicaulis (Schltdl. et Cham.) Mez.

Cinnamomum areolatum: Phoebe areolata Lundell

Cinnamomum breedlovei: Phoebe breedlovei Lundell

Cinnamomum chiapense: Phoebe chiapensis Lundell

Cinnamomum concinnum: Phoebe elegans van der Werff

Cinnamomum effusum: Phoebe effusa M eisn., Phoebe barbeyana M ez, Cinnamomum barbeyanum (M ez) Kosterm. Cinnamomum grisebachii: Phoebe triplinervis Griseb.

Cinnamomum pachypodum: Phoebe pachypoda (N ees) Mez, Phoebe benthamiana (N ees) Mez, Phoebe hartwegii M eisn., Phoebe arsenei C.K.Allen, Cinnamomum arsenei (C.K.Allen) Kosterm.

Cinnamomum triplinerve: Persea cinnamomifolia H.B.K., Phoebe cinnamomifolia (H.B.K.) Nees, Cinnamomum cinnamomifolium (H.B.K.) Kosterm., Phoebe mexicana M eisn., Persea mexicana (M eisn.) H emsl., Cinnamomum mexicanum (M eisn.) Kosterm., Phoebe mexicana var. bourgeauviana M ez.

Licaria campechiana: O cotea campechiana Standl., M isanteca campechiana (Standl.) Lundell, Acrodiclidium campechianum (Standl.) Lundell, Acrodiclidium Iucidum Lundell, Licaria Iucida (Lundell) C.K.Allen, M isanteca lucida (Lundell) Lundell

Licaria caudata: Chanekia caudata Lundell, Acrodiclidium caudatum (Lundell) Lundell

Licaria capitata: Misanteca capitata Cham. et Schltdl., Acrodiclidium capitatum (Cham. et Schltdl.) Lundell, Acrodiclidium glabrum Brandegee, Aniba mexicana Kosterm., Licaria oblongifolia Lundell

Licaria cervantesii: Laurus cervantesii H .B.K., Acrodiclidium cervantesii (H.B.K.) Lundell, M isanteca cervantesii (H.B.K.) Lundell

Licaria excelsa: Acrodiclidium excelsum (Kosterm.) Lundell, Misanteca excelsa (Kosterm.) Lundell, Licaria alata Miranda, Misanteca alata (Miranda) Miranda

Licaria mexicana: Acrodiclidium mexicanum Brandegee, Chanekia mexicana (Brandegee) Lundell, Misanteca mexicana (Brandegee) Lundell

Licaria misantlae: Acrodiclidium misantlae Brandegee, Chanekia misantlae (Brandegee) Lundell, Misanteca misantlae (Brandegee) Lundell

Licaria peckii: M isanteca peckii I.M .Johnst., Chanekia peckii (I.M .Johnst.) Lundell, Acrodiclidium peckii (I.M.Johnst.) Lundell

Licaria urceolata: Misanteca urceolata (Lundell) Lundell

Litsea glaucescens: Tetranthera glaucescens var. subsolitaria M eisn., Litsea glaucescens var. subsolitaria (M eisn.) Hemsl ., Litsea guatemalensis M ez, Litsea flavescens Bartlett, Litsea schaffneri Bartlett, Litsea acuminatissima Lundell, Litsea matudai Lundell, Litsea pallens Lundell, Litsea neesiana (Schauer) Hemsl., Litsea orizabae (M art. et Gal.) M ez

Nectandra ambigens: Phoebe ambigens S.F.Blake, Nectandra venosissima Lundell, O cotea venosissima (Lundell) Lundell

Nectandra cissiflora: Nectandra paulii C.K.Allen

Nectandra coriacea: Laurus coriacea Sw., O cotea lundellii Standl.

Nectandra cuspidata: Nectandra gentlei Lundell, Nectandra membranacea (Sw.) Spreng. ssp. cuspidata (N ees) Rohwer Nectandra hihua: Laurus hihua Ruiz et Pav., Nectandra glabrescens Bentham, Sassafridium macrophyllum Rose, Nectandra albiflora Lundell

Nectandra longicaudata: Phoebe longicaudata Lundell, Phoebe savannarum Standl. et Steyerm., N ectandra savannarum (Standl. et Steyerm.) C.K.Allen

Nectandra Iundellii: Persea gentlei Lundell, Phoebe gentlei (Lundell) Standl. et Steyerm.

Nectandra martinicensis: Nectandra tabascensis Lundell, O cotea tabascensis (Lundell) Howard

Nectandra matudai: Nectandra glandulosa Lundell, Pleurothyrium glandulosum (Lundell) Lundell

Nectandra membranacea: Laurus membranacea Sw., N ectandra skutchii C.K.Allen, Nectandra standleyi C.K.Allen

Nectandra nitida: Nectandra perdubia Lundell

Nectandra purpurea: Laurus purpurea Ruiz et Pav.

Nectandra reticulata: Laurus reticulata Ruiz et Pav.

Nectandra salicifolia: O cotea salicifolia H.B.K., Persea salicifolia (H.B.K.) Spreng., Nectandra sanguinea Rottboell var. lanceolata M eisn., Nectandra loesneri Mez, Nectandra cayoana Lundell

Nectandra turbacensis: $O$ cotea turbacensis H.B.K., Nectandra turbacensis (H.B.K.) N ees var. mexicana M eisn. 
O cotea acuminatissima: Phoebe acuminatissima Lundell, Cinnamomum acutatum Kosterm., Phoebe saxchanalensis Lundell, Phoebe siltepecana Lundell, Cinnamomum siltepecanum (Lundell) Kosterm.

o cotea bernoulliana: Phoebe glabra van der W erff, Cinnamomum vanderwerffii Kosterm., O cotea vanderwerffii (Kosterm.) van der Werff

O cotea botrantha: Persea matudai Lundell

O cotea cernua: O reodaphne cernua N ees

O cotea chiapensis: Nectandra chiapensis Lundell

0 cotea dendrodaphne: $O$ cotea ovandensis Lundell, O cotea quisara Mez et J.D.Smith

0 cotea effusa: $O$ reodaphne effusa M eisn.

O cotea helicterifolia: Oreodaphne helicterifolia M eisn., Phoebe helicterifolia (M eisn.) M ez, O reodaphne mexicana M eisn., O cotea mexicana (M eisn.) Hemsl., O reodaphne mexicana var. subsessilis M eisn., O cotea mexicana var. subsessilis (M eisn.) Hemsl., O reodaphne mexicana var. Iongipes M eisn., O cotea mexicana var. Iongipes (M eisn.) Hemsl., Phoebe nectandroides M ez

0 cotea klotzschiana: O reodaphne klotzschiana N ees

O cotea leucoxylon: O cotea lenticellata Lundell

O cotea magnifolia: Nectandra magnifolia Lundell, Nectandra thornei Lundell

O cotea platyphylla: Phoebe platyphylla Lundell, Nectandra platyphylla (Lundell) C.K.Allen

o cotea psychotrioides: O reodaphne psychotrioides(H.B.K.) N ees, Phoebe psychotriodes(H.B.K.) M ez, Cinnamomum psychotrioides (H.B.K.) Kosterm.

O cotea rubriflora: Nectandra rubriflora (Mez) C.K.Allen, O cotea perseifolia M ez et J.D.Smith

O cotea salvinii: Phoebe salvinii (M ez) Lundell, Cinnamomum salvinii (M ez) Kosterm.

O cotea sinuata: N ectandra sinuata M ez

O cotea standleyi: Phoebe macrophylla Standl. et Steyerm.

O cotea veraguensis: $O$ cotea bakeri S.F.Blake, O cotea escuintlensis Lundell

Persea albida: Persea guatemalensis Lundell

Persea americana: Persea drymifolia Cham. et Schlecht., Persea floccosa M ez, Persea gigantea L.O.W illiams, Persea nubigena L.O.Williams

Persea liebmannii: Persea podadenia S.F.Blake, Persea flavifolia Lundell, Persea longipes (Schltdl.) M eisn.

Persea schiedeana: Persea gratissima var. schiedeana (N ees) M eisn.

Persea vesticula: Persea chiapensis Lundell, Persea popenoei L.O .W illiams 